\title{
A sulfur-sulfur cross-linked polymer synthesized from a polymerizable dithiocarbamate as a source of dormant radicals
}

\author{
Luis Miguel García-Con, Michael J. Whitcombe*, Elena V. Piletska and Sergey A. Piletsky \\ Cranfield Health, Cranfield University, Vincent Building, Cranfield, Bedfordshire, MK43 OAL, UK \\ Fax: (+44) 1234758380 E-mail: m.whitcombe@cranfield.ac.uk
}

Keywords: radical reactions $\cdot$ reversible cross-linking $\cdot$ thiuram $\cdot$ photochemistry $\cdot$ dithiocarbamate ester

\begin{abstract}
The dithiocarbamates (DTCs) have a special place within polymer chemistry due to their versatile role as thermal and photochemical initiators.[1] In particular the role of dithiocarbamate esters as "iniferters" in "living" radical polymerizations has practical significance.[2] The living nature of DTC ester-initiated photopolymerization derives from the extreme difference in reactivity of the radicals created by homolytic cleavage of the DTC ester bond. This gives rise to a reactive carbon-centred radical and a much less reactive, or dormant, dithiocarbamate radical, in which the unpaired electron is delocalized over the DTC structure. The chemistry of the latter is dominated by recombination to reform covalent bonds. It is the ability of dormant radicals to recapture the carbon-centred radical after addition of monomer to form a new DTC ester that imparts living character to DTC ester-initiated photopolymerization. Compounds of this type have been used in the synthesis of telechelic polymers,[3] block[4,5] or graft copolymers[5,6] and hyperbranched polymers.[7] In the latter context, Otsu et al.[8] synthesized the first example of a DTC ester of a polymerizable derivative; the 4-vinylbenzyl ester of $N, N$-diethyldithiocarbamic acid, creating "inimer" - a compound that combines iniferter and monomer functionalities. Inimer could be polymerized photochemically to form hyperbranched polymers or by conventional thermal initiation to form linear polymers with pendant DTC ester groups. These polymers are macro-iniferters, which allow the photochemical grafting of a range of other monomers. As well as the inimer of Otsu,[8] similar compounds, based on methacrylates, have been reported.[9]

In polymers of these compounds, irradiation will generate active macroradicals and dormant dithiocarbamyl radicals as low molar mass fragments. The macroradicals will react rapidly with monomer, oxygen or with dithiocarbamyl radical to reform DTC esters. The situation will be different however if the dithiocarbamyl radical is part of the polymeric system and the active radical is the low molar mass fragment. A likely scenario under these circumstances is that during prolonged irradiation, the more readily diffusible carbon-centred radicals will dimerise, forming stable low-molar mass by-products, leading to an excess of dithiocarbamyl radicals attached to the polymer. This will result in the creation of a relatively stable population of polymeric dormant radicals. The physics and chemistry of immobilized dithiocarbamyl dormant radicals is expected to be an exciting field of investigation, since materials containing stable radicals may possess useful photo-responsive magnetic, mechanical and electrical properties. Polymers with other stable radical functionalities, such as nitroxy radicals, have been reported as novel battery materials[10] and alkoxamines in the side chain[11] or main chain[12] and have been used as thermally-activated systems for the reorganization of polymeric materials, via radical crossover reactions. Similarly, reversible decross-linking to radical fragments has been shown to occur in response to light or pressure, in the case of triphenylimidazole dimers, present as crosslinks on a methacrylate backbone.[13]

In order to develop new materials to explore the properties of polymeric dithiocarbamyl radicals, we designed a monomer, superficially similar to inimer, [8] but reversing the orientation of the molecule, as shown in Scheme 1, such that the dithiocarbamyl residue is the polymerizable part of the molecule.
\end{abstract}

The monomer, 1 (Scheme 1) was prepared by reaction of $\mathrm{CS}_{2}$ with amine in the presence of base,[14] following the "onepot" method proposed by Azizi et al.[15] A nominally linear polymer, (2) was prepared in toluene by AIBN-initiated thermal polymerization in the dark.

UV irradiation of solutions of $\mathbf{2}$ is expected to result in cleavage of the C-S bond, creating benzyl radicals and polymeric sulfur radicals. While recombination of the radical pair will reform the initial DTC ester groups, recombination of "like" radicals will result in cross-linking due to the formation of S-S bonds and, as frustrations due to cross-linking increase, isolated sulfur radicals, which are expected to be relatively long-lived. This process will be aided by benzyl radical recombination to form 1,2diphenylethane (4) as by-product, (Scheme 2). Irradiation of 2 was performed in chloroform solution. While this solvent is not ideal for performing photochemical studies, due to the possibility of side-reactions from photolysis of the solvent, nevertheless it was used on account of the greater solubility of the polymer in $\mathrm{CHCl}_{3}$ than in other solvents (such as acetonitrile) which do not suffer from these problems.

The kinetics of radical formation during irradiation of $\mathbf{1}$ and $\mathbf{2}$ was investigated by EPR (Figure 1a). In these two cases the DTC ester group is intact at the start of the experiment. The intensity of the EPR signal was seen to increase linearly as a function of irradiation time over the first $2000 \mathrm{~s}$, starting from zero intensity at time $t=0 \mathrm{~s}$. This is a characteristic of a system in which the formation of active species is fast compared to other processes (such as polymer propagation) and where the rate of termination 
can be considered to be negligible until relatively high conversions.[16] The auto cross-linked polymer, 3, showed different behaviour, possessing a significant EPR signal before irradiation due to the presence of isolated dormant radical species (Figure 1b). Since this sample had been prepared more than 2 weeks previously, it shows the remarkable longevity of dormant radicals in this system. The generation of additional radical species is expected to occur as a result of dissociation of thiuram groups, present as cross-links between polymer chains. The rate of development of the EPR signal in the case of $\mathbf{3}$ was not linear with time. The signal did not increase after $\sim 1500 \mathrm{~s}$, suggesting that the rates of radical formation and loss were approximately equal.

In order to investigate the early stages of photochemical cross-linking, samples of 2 were irradiated for 1, 3 or 5 min only and the solutions examined by GPC. The non-irradiated 2 (Figure 2a, (i)) showed a monomodal peak centred at 18k. During irradiation, the solution viscosity increased rapidly due to increases in molar mass. The average MW of the polymeric fraction produced after $5 \mathrm{~min}$ irradiation was $20 \mathrm{k}$ (Figure $2 \mathrm{~b}$, upper line). The solution also contained a growing fraction with a much higher MW, estimated to be $>150 \mathrm{k}$, which is most likely formed during the initial stages of gel formation.

$\mathrm{UV}$ irradiation for around $4 \mathrm{~h}$ was required to create 3 which was no longer soluble in either $\mathrm{CHCl}_{3}$ or THF and appeared as a swollen gel. Precipitation into methanol was performed to separate the gel fraction from soluble material and the supernatant analysed by GPC. The result, (Figure 2a, curve ii) showed complete disappearance of 2 (MW $=18 \mathrm{k}$ ) and formation of both high and low molecular weight products, proving that cross-linking had occurred between polymer chains. The by-product of active radical recombination, 1,2-diphenylethane (4) was also detected, the peak in the GPC having the same retention time as an authentic sample. Identification of the by-product was confirmed by HPLC-MS and NMR. Evidence for the proposed mechanism of cross-linking can also be seen in the FT-IR. The spectrum of $\mathbf{3}$ (Figure 3, upper curve) showed a strong peak due to S-S stretching at $750 \mathrm{~cm}^{-1}$, [17] which was absent from the spectra of 1 and $\mathbf{2}$ (Figure 3, lower curve). Signals due to C=S and C-N stretching vibrations, at 1174 and $1477 \mathrm{~cm}^{-1}$ respectively, were found in the spectra of all three compounds.

As a final demonstration that the cross-links in $\mathbf{3}$ were due to reversible formation of S-S bonds, $\mathbf{3}$ was irradiated with an excess of tetraethyl dithiuram disulfide (6), (Scheme 3 ). Under these conditions the polymer was rendered completely soluble after $6 \mathrm{~h}$ irradiation, followed by $42 \mathrm{~h}$ in the dark, the GPC (Figure 4) and NMR (Supporting Information, Figure S2) being consistent with the change.

We have presented an efficient approach to the synthesis of a new range of photoresponsive DTC-containing polymeric materials. We believe that the ability to generate a high concentration of stable radicals under controlled conditions has the potential to be used in many application areas. These may include engineering adhesives, UV filters, separation materials, sensors for radicals, trapping of reactive oxygen species and as antioxidants, thermoplastic vulcanizates, and as new materials for microelectronics.

\section{Experimental Section}

Details of the synthesis of all compounds and polymers and of the irradiation experiments are given in the supporting information.

Received: ((will be filled in by the editorial staff))

Published online on ((will be filled in by the editorial staff))

\section{Acknowledgements}

We acknowledge Cranfield Health for funding and thank Bruker Biospin, Germany for providing the EPR data.

Supporting information for this article is available on the WWW under http://www.angewandte.org or from the author.

[1] a) R. J. Kern, J. Am. Chem. Soc. 1955, 77 1382-1383; b) T. E. Ferington, A. V. Tobolsky, J. Am. Chem. Soc. 1955, 77 4510-4512; c) T. Otsu, K. Nayatani, Makromol. Chem. 1958, 27 149-156; d) T. Otsu, K. Nayatani, I. Muto, M. Imai, Makromol. Chem. 1958, 27 142-148; e) C. P. Reghunadhan Nair, G. Clouet, Polymer 1988, 29 1909-1917.

[2] T. Otsu, J. Polym. Sci. Part A: Polym. Chem. 2000, 38 2121-2136.

[3] R. Jerome, M. Henrioulle-Granville, B. Boutevin, J. J. Robin, Prog. Polym. Sci. 1991, 16 837-906.

[4] T. Otsu, A. Kuriyama, Polym. J. 1984, 17 97-104.

[5] T. Otsu, J. Polym. Sci. 1957, 26 236-239.

[6] P. K. Bhuyan, D. K. Kakati, J. Appl. Polym. Sci. 2009, 112 64-71.

[7] K. Ishizu, A. Mori, Macromol. Rapid Commun. 2000, 21 665-668.

[8] T. Otsu, K. Yamashita, K. Tsuda, Macromolecules 1986, 19 287-290.

[9] a) S. H. Qin, K. Y. Qiu, Eur. Polym. J. 2001, 37 711-717; b) N. Luo, J. B. Hutchison, K. S. Anseth, C. N. Bowman, J. Polym. Sci. Part A: Polym. Chem. 2002, 40 1885-1891.

[10] T. Suga, H. Konishi, H. Nishide, Chem. Commun. 2007, 1730-1732.

[11] Y. Higaki, H. Otsuka, A. Takahara, Macromolecules 2006, 39 2121-2125.

[12] a) H. Otsuka, K. Aotani, Y. Higaki, A. Takahara, Chem. Commun. 2002, 2838-2839; b) H. Otsuka, K. Aotani, Y. Higaki, A. Takahara, J. Am. Chem. Soc. 2003, 125 4064-4065.

[13] T. Iwamura, M. Sakaguchi, Macromolecules 2008, 41 8995-8999.

[14] W. D. Rudorf, J. Sulf. Chem. 2007, 28 295-339.

[15] N. Azizi, F. Aryanasab, M. R. Saidi, Org. Lett. 2006, 8 5275-5277. 
[16] J. Merna, J. Cihlar, M. Kucera, A. Deffieux, H. Cramail, Eur. Polym. J. 2005, 41 303-312.

[17] A. Patel, K. Mequanint, J. Polym. Sci. Part A: Polym. Chem. 2008, 46 6272-6284. 


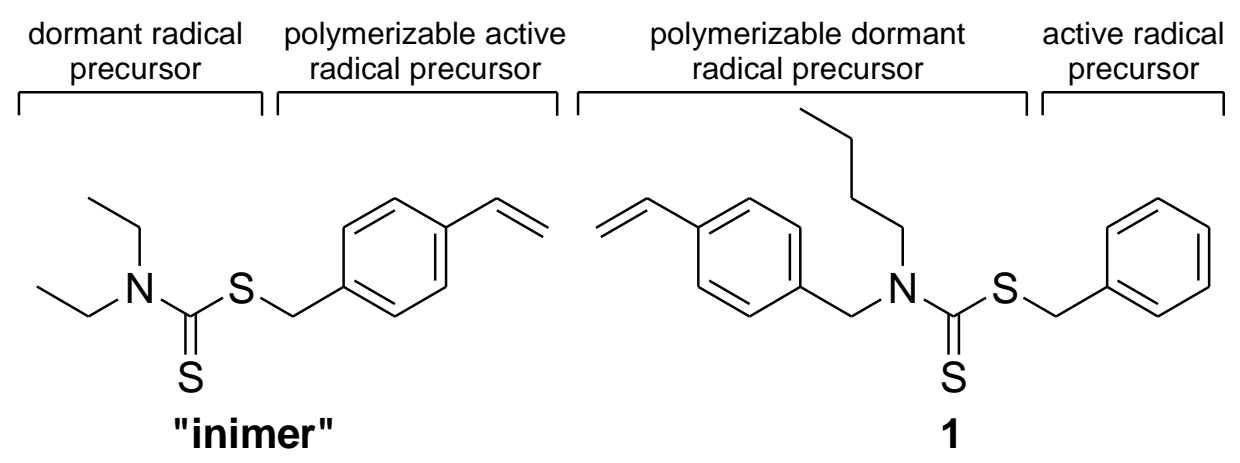

Scheme 1. The structure of Otsu's "inimer"[8] (left) and of the monomer used in this work: benzyl $N$-butyl- $N$-(4-vinylbenzyl) dithiocarbamate, (1), or "reversed inimer" (right), showing the differences between the two structures with respect to the active and dormant radical precursor sites and the position of the polymerizable functionality. 
<smiles>CCCCN(Cc1ccc(C(C)(C)C)cc1)C(=S)SCc1ccccc1</smiles>

2

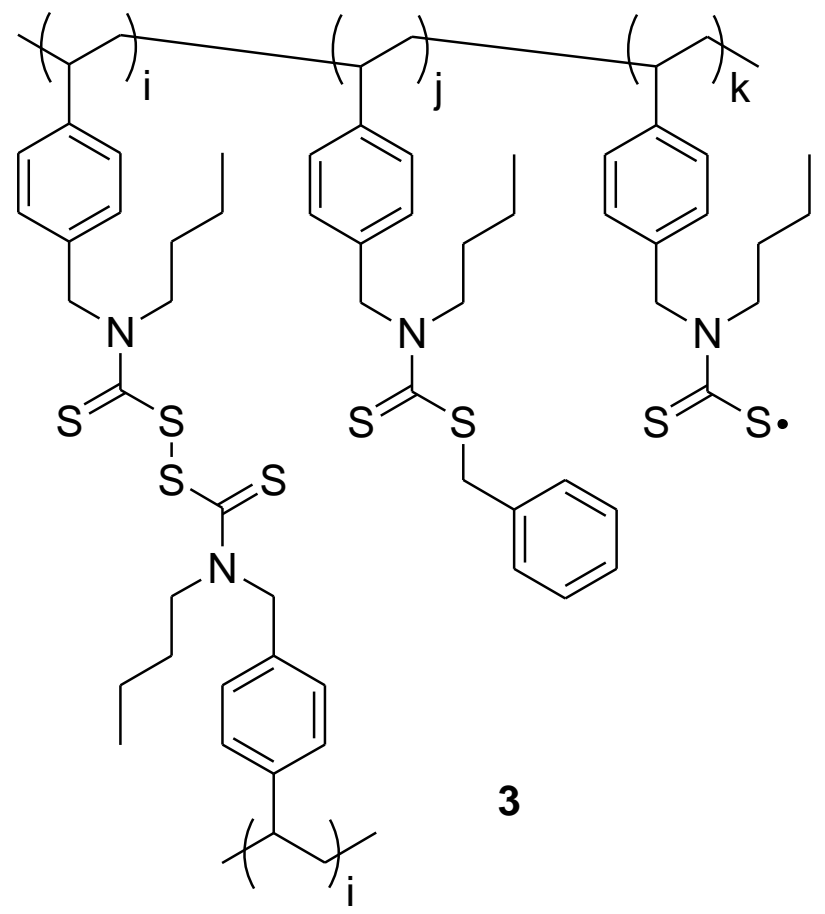

Scheme 2. Photocross-linking of 2 to 3 , with elimination of 1,2-diphenylethane (4). 
a)

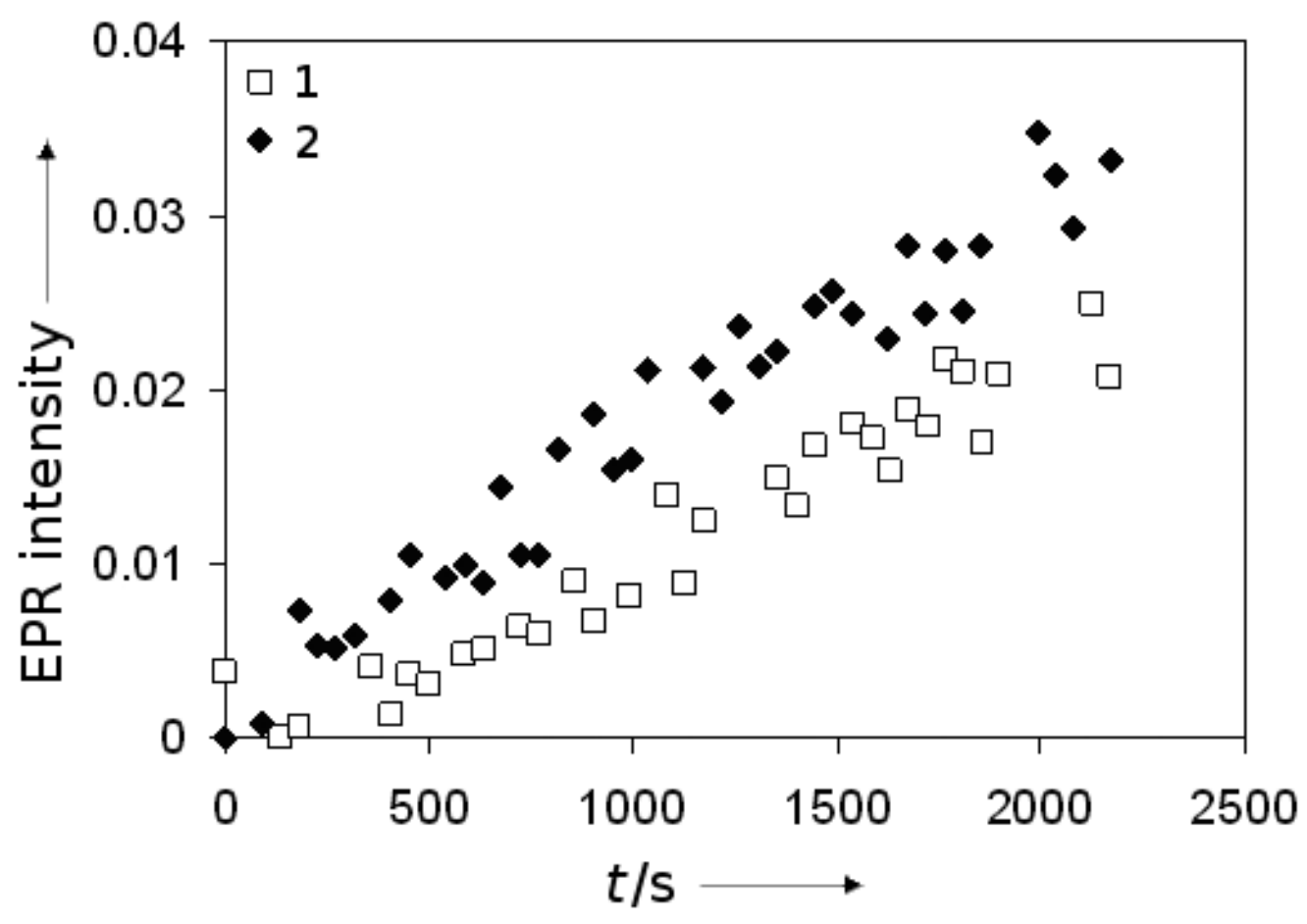

b)

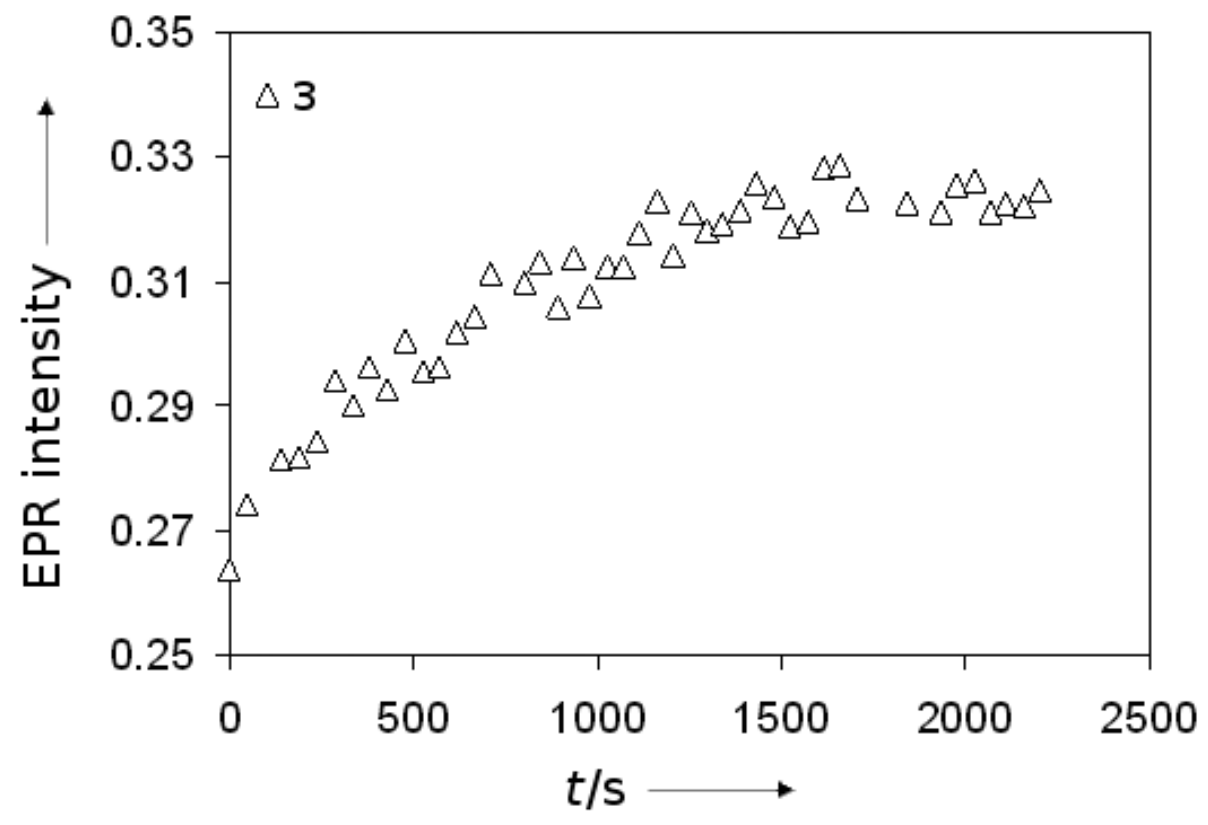

Figure 1. EPR graphs showing the intensity of the EPS signal against irradiation time for: a) $\mathbf{1}$ and $\mathbf{2}$; and b) 3 . 

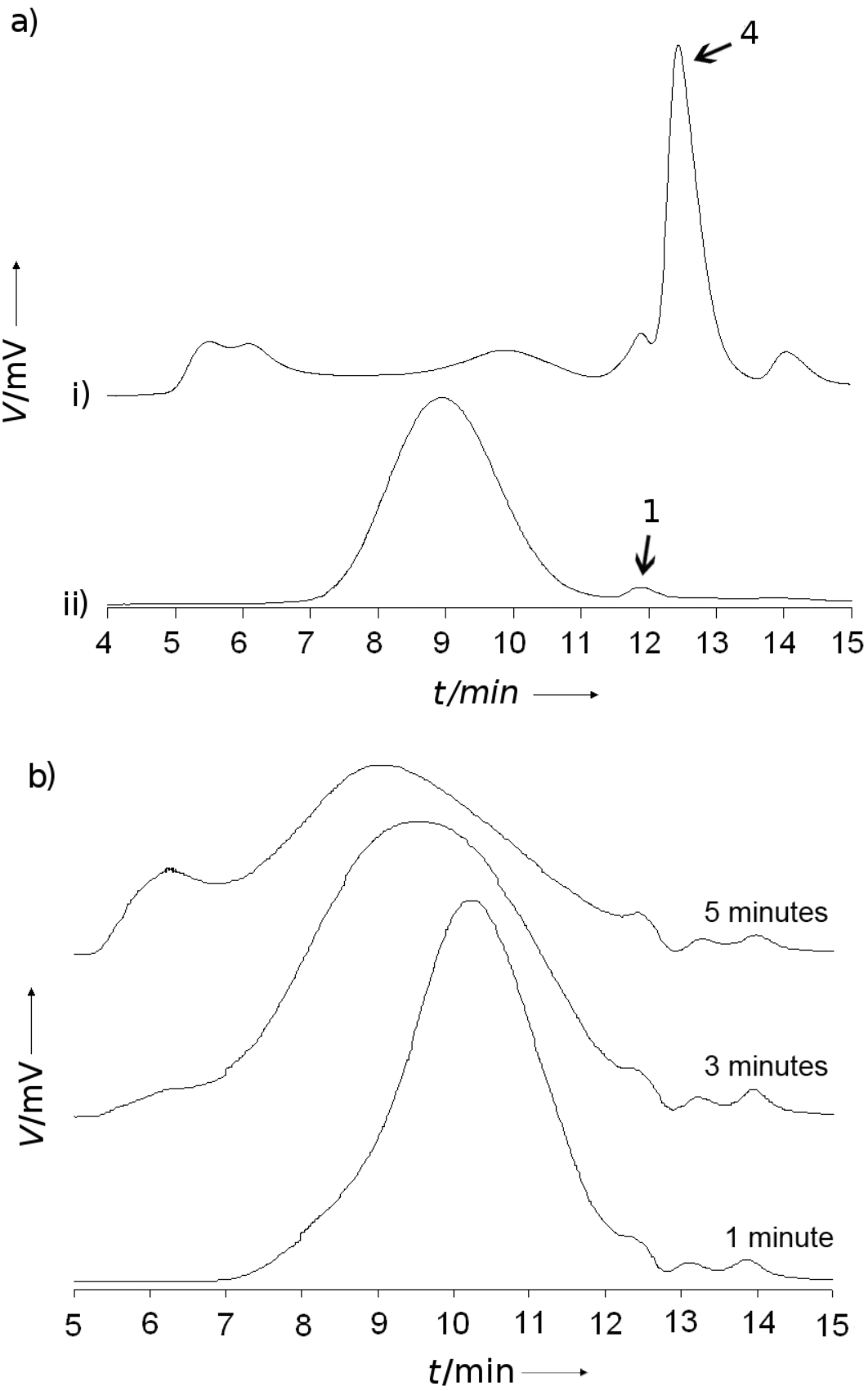

Figure 2. GPC curves for: a) 2 (i) and $\mathbf{3}$ supernatant (ii); b) samples taken from the initial stages of the photochemical crosslinking reaction. 


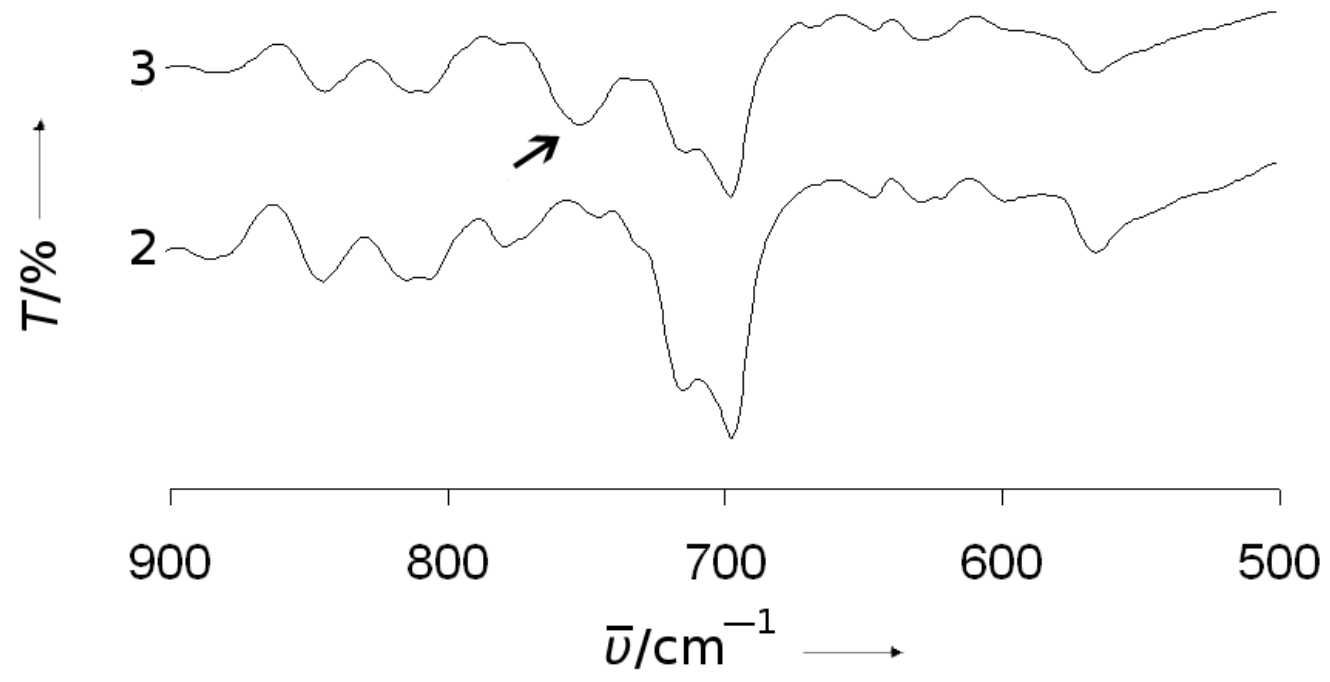

Figure 3. IR spectra of the linear (2) and cross-linked polymers (3), showing the appearance of a new band due to S-S bond formation. 

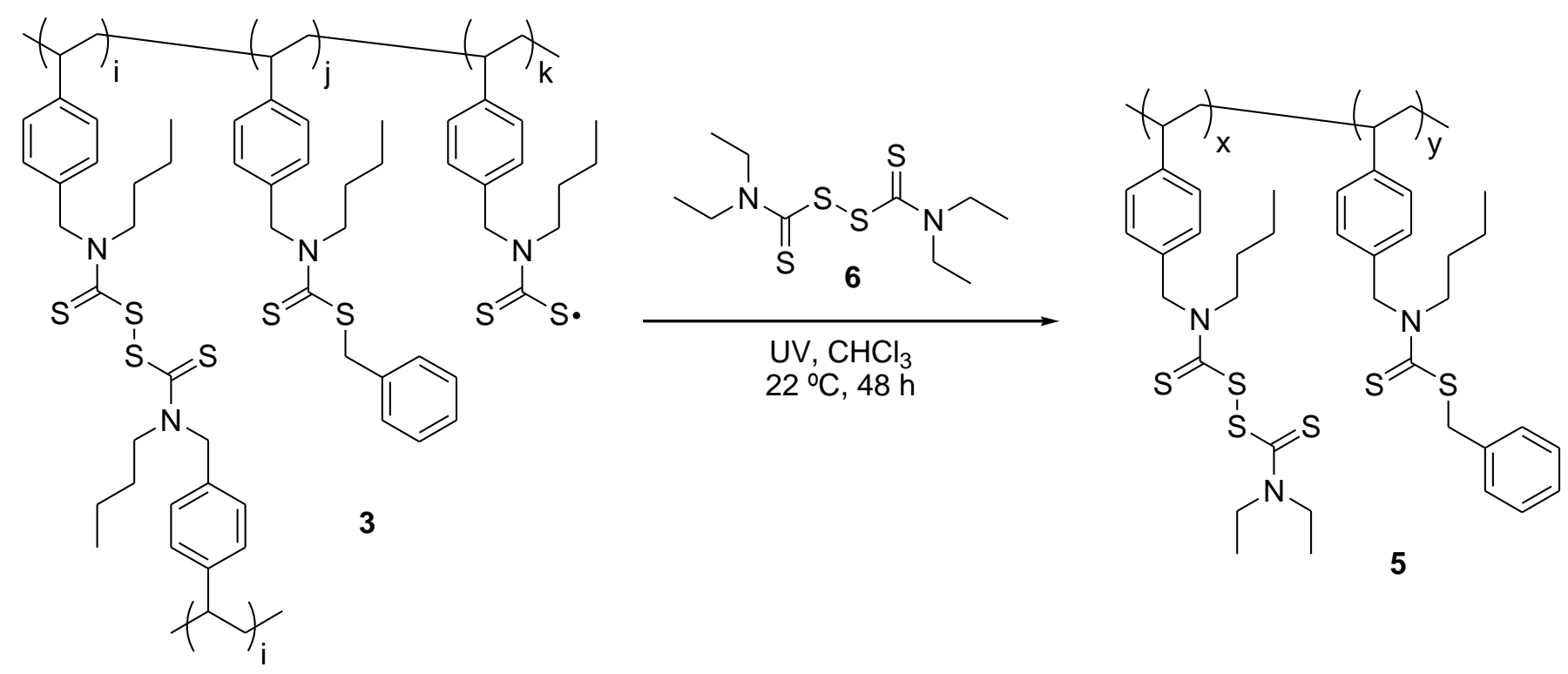

Scheme 3. Decross-linking of 3 (to 5 ) by photochemical treatment with tetraethyl dithiuram disulfide (6). 


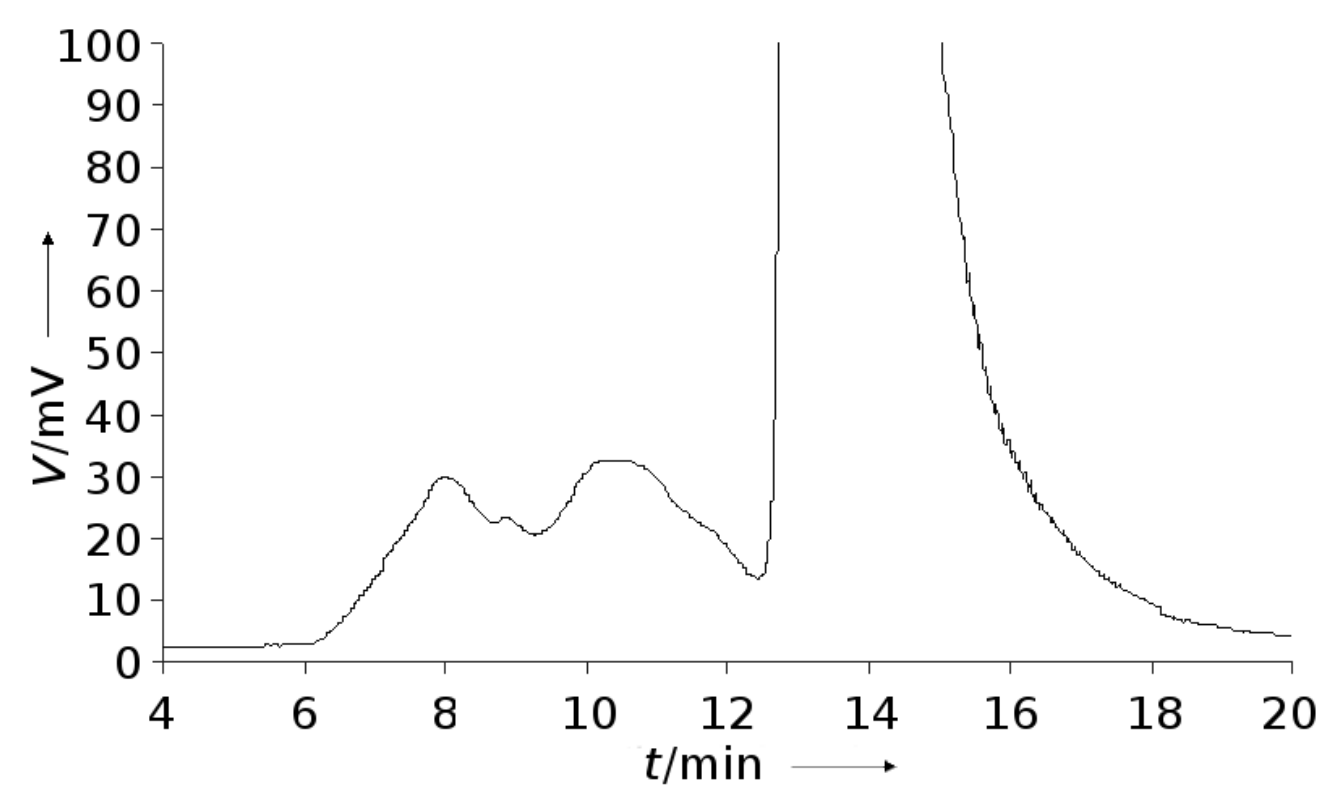

Figure 4. GPC of 5, showing reformation of soluble polymeric products. 